\title{
nature Siructural
}

\section{Where oxygen is scarce}

$\mathrm{T}$ here has been much celebration this year to mark the passing of a half century since the double helical structure of DNA was put forward. But 2003 is notable in another sense, of course; this year marks the $50^{\text {th }}$ anniversary of the conquering of Mount Everest by Edmund Hillary and Tenzing Norgay. Although you will not see extensive coverage of that accomplishment in the pages of Nature Structural Biology — we'll leave that to National Geographic - it does call to mind a topic of considerable recent interest to structural and molecular biologists, the oxygen-sensing pathway and consequent physiological response to oxygen deprivation.

Oxygen deprivation, or hypoxia, triggers a physiological response mediated by the key transcription factor hypoxiainducible factor-1 (HIF-1). HIF-1 is a heterodimer consisting of the unstable HIF-1 $\alpha$ subunit and a constitutively expressed HIF-1 $\beta$ subunit. When physiologically normal oxygen levels are present, HIF- $1 \alpha$ is targeted for destruction. However, as a consequence of lower than normal oxygen levels, HIF- $1 \alpha$ is stabilized and transported from the cytoplasm to the nucleus, where it dimerizes with HIF-1 $\beta$. The intact HIF-1 heterodimer then binds to gene promoters containing hypoxia-responsive elements, activating the transcription of gene products associated with oxygen homeostasis and adaptation to hypoxia.

The details of the oxygen-sensing pathway started to come into focus early in 2001, with independent reports from the groups of William Kaelin and Peter Ratcliffe aimed at understanding the regulatory mechanism behind HIF-1 $\alpha$ degradation. These studies identified a specific post-translational modification, hydroxylation of a conserved proline residue within HIF-1 $\alpha$. This modified proline is recognized by the product of the von Hippel-Lindau tumor suppressor gene, pVHL, which tags the $\alpha$ subunit for degradation by the proteasome. The enzymes responsible for the post-translational modification of HIF- $1 \alpha$ belong to a family of iron-dependent prolyl hydroxylases that employ $\mathrm{O}_{2}$ as a substrate in the hydroxylation reaction. Thus, these enzymes seem to provide the direct link between oxygen levels and HIF-1 function.

While this all seems relatively straightforward, more recent findings have added additional levels of complexity. Researchers have identified a second modification, the hydroxylation of an asparagine residue in the HIF- $1 \alpha \mathrm{C}$-terminal transactivation domain. This modification disrupts the interface with the cysteine-histidine rich domain of transcriptional coactivators, such as CBP and p300, providing an additional oxygen-sensing switch which may serve as a failsafe mechanism.

A report by Michael Eck and co-workers on page 504 of this issue now adds another piece to the puzzle. Eck and co-workers report the structure of a complex formed between the cysteinehistidine rich domain of p300 and the transactivation domain of CITED2, a putative negative regulator of HIF-1 $\alpha$. The presence of three tandem hypoxia-responsive elements in the CITED2 gene promoter suggests that CITED2 expression is upregulated during hypoxia. Eck and co-workers demonstrate that CITED2 disrupts a complex between the transactivation domain of HIF- $1 \alpha$ and p300's cysteine-histidine rich domain. Their studies suggest a mechanism whereby CITED2 regulates HIF-1 by competing for a single binding site on p300, a control mechanism that could be involved in shutting down the hypoxia response when oxygen levels have returned to normal.

In addition to mediating the hypoxia response, HIF- 1 activity is also critical for related physiological events, including blood vessel formation during embryogenesis, as well as pathophysiological processes such as the formation of angiogenic tumors. This link to cancer, and the involvement of HIF-1 in diabetes and a variety of ischemic diseases, should make HIF-1 an attractive drug target. But determining effective therapeutic strategies will depend on a thorough understanding of the finer points of the oxygen-sensing pathway. This comprehensive understanding will no doubt be achieved in much the same way that Hillary and Norgay ultimately conquered Mount Everest_-one step at a time. 